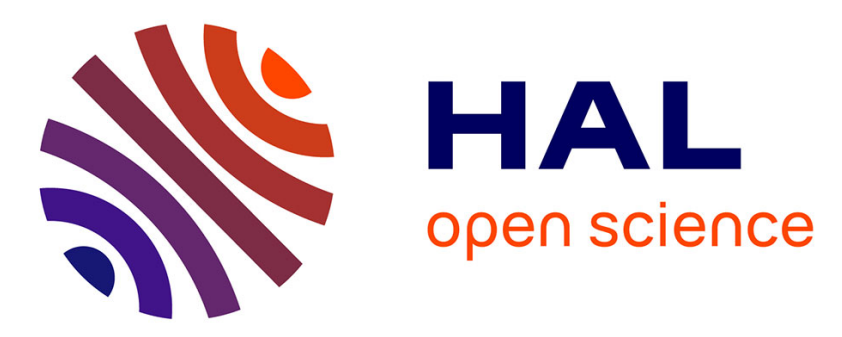

\title{
Characterization of myocardial motion by multiple kernel learning: application to heart failure with preserved ejection fraction
}

Sergio Sanchez-Martinez, Nicolas Duchateau, Bart Bijnens, Tamás Erdei, Alan Fraser, Gemma Piella

\section{To cite this version:}

Sergio Sanchez-Martinez, Nicolas Duchateau, Bart Bijnens, Tamás Erdei, Alan Fraser, et al.. Characterization of myocardial motion by multiple kernel learning: application to heart failure with preserved ejection fraction. 8th International Conference, FIMH 2015, Maastricht, The Netherlands, June 25-27, 2015. Proceedings, 2015, Maastricht, Netherlands. pp.65-73, 10.1007/978-3-319-20309-6_8 . hal-01208016

\section{HAL Id: hal-01208016 https://hal.inria.fr/hal-01208016}

Submitted on 1 Oct 2015

HAL is a multi-disciplinary open access archive for the deposit and dissemination of scientific research documents, whether they are published or not. The documents may come from teaching and research institutions in France or abroad, or from public or private research centers.
L'archive ouverte pluridisciplinaire HAL, est destinée au dépôt et à la diffusion de documents scientifiques de niveau recherche, publiés ou non, émanant des établissements d'enseignement et de recherche français ou étrangers, des laboratoires publics ou privés. 


\title{
Characterization of Myocardial Velocities by Multiple Kernel Learning: Application to Heart Failure with Preserved Ejection Fraction
}

\author{
Sergio Sanchez-Martinez ${ }^{1}$, Nicolas Duchateau ${ }^{3}$, Bart Bijnens ${ }^{1,2}$, Tamás Erdei $^{4}$, \\ Alan Fraser ${ }^{4}$, and Gemma Piella ${ }^{1}$ \\ 1 DTIC, Universitat Pompeu Fabra, Barcelona, Spain \\ ${ }^{2}$ ICREA, Barcelona, Spain \\ 3 Asclepios Research Project, INRIA Sophia Antipolis, France \\ ${ }^{4}$ Wales Heart Research Institute, Cardiff University, UK
}

\begin{abstract}
The present study aims at improving the characterization of myocardial velocities in the context of heart failure with preserved ejection fraction (HFPEF) by combining multiple descriptors. It builds upon a recent extension of manifold learning known as multiple kernel learning (MKL), which allows the combination of data of different natures towards the learning. Such learning is kept unsupervised, thus benefiting from all the inherent explanatory power of the data without being conditioned by a given class. The methodology was applied to $2 \mathrm{D}$ sequences from a stress echocardiography protocol from 33 subjects (21 healthy controls and $12 \mathrm{HFPEF}$ subjects). Our method provides a novel way to tackle the understanding of the HFPEF syndrome, in contrast with the diagnostic issues surrounding it in the current clinical practice. Notably, our results confirm that the characterization of the myocardial functional response to stress in this syndrome is improved by the joint analysis of multiple relevant features.
\end{abstract}

\section{Introduction}

Heart failure with preserved ejection fraction (HFPEF) is recognized as a public health problem of growing concern. This syndrome presents signs of heart failure but still maintains the ejection fraction into a normal range. Left ventricular diastolic dysfunction is a leading mechanism causing the syndrome [9] but recent studies suggest that HFPEF is a rather heterogeneous condition consisting of several pathophysiological subtypes [7]. Due to this heterogeneity, there is still an incomplete understanding of the syndrome, which results in a low statistical power of current diagnostic methods [10].

Previous attempts to characterize HFPEF [9] were mainly based on relatively simple measurements taken at rest, such as ejection fraction or E/e' (ratio of the early transmitral flow velocity by pulsed Doppler and the early mitral annular velocity by myocardial velocity imaging). Myocardial velocity imaging (MVI) provides a direct measurement of the velocities of the myocardial tissue with 


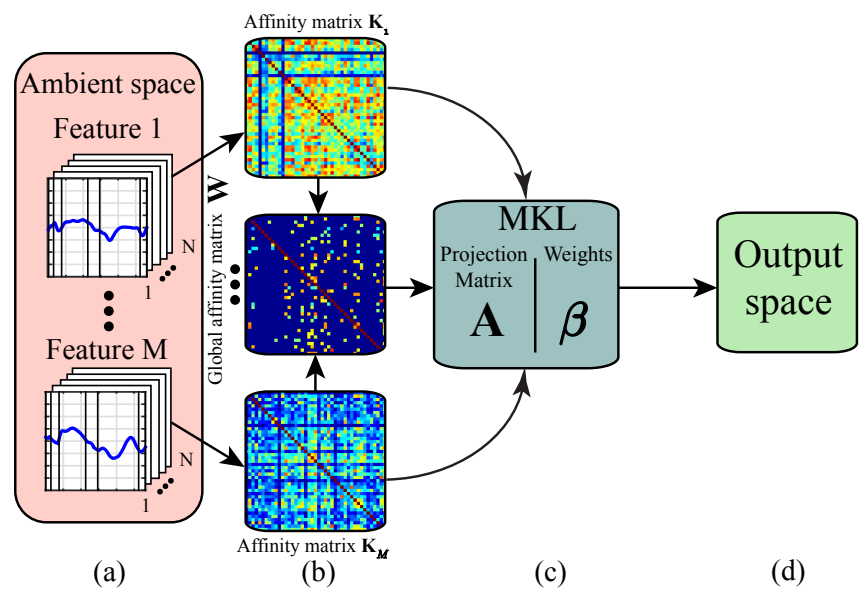

Fig. 1: Pipeline of the proposed method: (a) ambient space with $M$ input features for each subject, (b) feature-specific and global affinity matrices used as input to the MKL optimization, (c) MKL optimization (d) Output space of reduced dimensionality.

high temporal resolution. However, they fail at summarizing the complexity of the observable abnormalities. In order not to discard useful information, further analysis on the data would be helpful.

We propose to take advantage of the full myocardial velocity traces (along the whole cardiac cycle), from two stages of a stress echocardiography protocol, to determine the diagnostic features of the disease. Functional atlases [2] provide a common spatiotemporal system of coordinates where such patterns can be compared. However, their analysis is often limited to linear statistical comparisons, or to voxel-wise observations. Non-linear dimensionality reduction techniques such as graph embedding [11] have been investigated to overcome these limitations and perform pattern-wise comparisons [3]. Nonetheless, such analyses only focus on single descriptors to characterize a given population in an unsupervised way (e.g. myocardial velocity in [3]). Depending on the complexity of the disease, a single descriptor strategy may not be sufficient to accomplish a proper characterization. In the present paper, we build upon a recently proposed framework, known as multiple kernel learning (MKL), which allows to optimally fuse heterogeneous information. A supervised learning formulation of MKL was given in [8].

Our main contribution is to adapt this formulation to an unsupervised setting suitable for our problem. We build upon the assumption that symptoms related to HFPEF will become apparent during exercise [4] and will be reflected in the myocardial velocity patterns especially of left ventricular long-axis function. Accordingly, the inputs for the MKL algorithm come from MVI acquisitions of healthy and suspected HFPEF subjects, at different stages of a stress protocol and in different regions of the left ventricular wall. 
We demonstrate the relevance of jointly analyzing these multivariate data within a MKL framework. Notably, clusters can be intuited from the velocity traces and agree with clinical diagnosis. Additionally, we show that the analysis is effectively improved by the inclusion of multiple features. These results suggest that the characterization of the HPFEF syndrome can be improved using our representation.

\section{Methods: unsupervised MKL for dimensionality reduction}

In the following, we describe how the MKL-based algorithm of [8] can be adapted to an unsupervised setting. Fig. 1 outlines the pipeline of the proposed algorithm.

The starting point is the ambient space containing the input data, organized in a set of $M$ features $\mathbf{x}_{m}, m \in[1, M]$. In the first step of MKL, a kernel-based affinity matrix $\mathbf{K}_{m}$ associated to each feature $\mathbf{x}_{m}$ is computed. We choose Gaussian kernels of the form: $\mathbf{K}_{m}\left(\mathbf{x}_{m i}, \mathbf{x}_{m j}\right)=\exp \left(-\left\|\mathbf{x}_{m i}-\mathbf{x}_{m j}\right\|^{2} / 2 \sigma_{m}^{2}\right)$, where $\mathbf{x}_{m i}$ stands for the feature $m$ of subject $i(i \in[1, N])$ and $\sigma_{m}$ for the kernel bandwidth, whose choice is discussed in Sec.3.2. Additionally, a sparse global affinity matrix $\mathbf{W}$ (each of its elements $W_{i j}$ codifying neighborhood membership) is calculated as $\mathbf{W}=\sum_{m=1}^{M} \hat{\mathbf{K}}_{m}$, where $\hat{\mathbf{K}}_{m}$ corresponds to the kernel $\mathbf{K}_{m}$ normalized by its variance. This serves to avoid that higher variability features have a larger impact in the neighborhood decision.

$\mathbf{W}$ and $\mathbf{K}_{m}$ are the inputs to the MKL block, which is the core of the algorithm. It is based on a Laplacian formulation [1] that ensures that subjects with similar characteristics (neighbors) remain close in the output space. In univariate graph embedding [11], the optimal embedding is obtained through the minimization of:

$$
\min _{\mathbf{v}} \sum_{i, j=1}^{N}\left\|\mathbf{v}^{\top} \mathbf{x}_{i}-\mathbf{v}^{\top} \mathbf{x}_{j}\right\|^{2} \mathbf{W}_{i, j}, \quad \text { s. t. } \sum_{i=1}^{N}\left\|\mathbf{v}^{\top} \mathbf{x}_{i}\right\|^{2} \mathbf{D}_{i, i}=1,
$$

where $\mathbf{D}$ is a diagonal matrix, whose values are the result of a row-wise summation of $\mathbf{W}, N$ is the number of subjects, $\mathbf{x}_{i}$ is the value of the one and only descriptor (univariate setting) associated to subject $i$ and $\mathbf{v}$ is the matrix that maps the input data into the output space. Notice that the values $W_{i j}$ for neighbors are high, thus forcing their proximity in the embedding.

We adapt this formulation to combine multiple features in an unsupervised MKL framework [8], through the minimization of:

$$
\begin{aligned}
\min _{\mathbf{A}, \boldsymbol{\beta}} & \sum_{i, j=1}^{N}\left\|\mathbf{A}^{\top} \mathbb{K}^{(i)} \boldsymbol{\beta}-\mathbf{A}^{\top} \mathbb{K}^{(j)} \boldsymbol{\beta}\right\|^{2} \mathbf{W}_{i, j} \\
\text { s.t. } & \sum_{i=1}^{N}\left\|\mathbf{A}^{\top} \mathbb{K}^{(i)} \boldsymbol{\beta}\right\|^{2} \mathbf{D}_{i, i}=1, \quad \beta_{m} \geq 0, \forall m \in[1, M],
\end{aligned}
$$


where $\mathbb{K}^{(i)}$ is defined for subject $i$ as $\mathbb{K}^{(i)}=\left[\mathbf{K}_{m}(n, i)\right]_{(n, m) \in[1, N] \times[1, M]}$.

The unknowns in Eq.2 are the matrix $\mathbf{A}$, which performs the final mapping to the output space, and the weights given to the different features $\boldsymbol{\beta}=$ $\left[\beta_{1} \ldots \beta_{M}\right]^{\top}$.

These values are calculated by means of a two-stage optimization strategy. The first stage of the optimization aims at optimizing $\mathbf{A}$, while $\boldsymbol{\beta}$ is fixed. This step is initialized by fixing $\beta_{m}=1, \forall m \in[1, M]$. The problem turns out to be a generalized eigenvalue problem, with an explicit solution. The second stage of the optimization aims at optimizing $\boldsymbol{\beta}$, while fixing the previously calculated A. This problem can be solved by quadratically constrained quadratic programming (QCQP), which is not straightforward. Nevertheless, it can be relaxed to a semidefinite programming problem, which can be solved more efficiently. In practice, this is addressed by the use of CVX, a package for solving convex programs [6]. Further details about the optimization can be found in [8].

The input samples are mapped to the output space by $\mathbf{y}=\mathbf{A}^{\top} \sum_{m=1}^{M} \mathbf{K}_{m} \beta_{m}$, where $\mathbf{y}=\left[\mathbf{y}_{1} ; \mathbf{y}_{2} ; \ldots ; \mathbf{y}_{N}\right]$ gathers (row-wise) the coordinates of each input sample in the output space.

\section{Experiments and results}

\subsection{Echocardiographic data}

The method was applied to $2 \mathrm{D}$ sequences in a 4-chamber view from a stress echocardiography protocol using a semi-supine bicycle. Commercial software (EchoPAC, v.113, GE Healthcare, Milwaukee, WI) was used for information extraction. The database consisted of 33 subjects (21 healthy controls and 12 HFPEF subjects, diagnosed by Paulus' criteria [9]), with age $68 \pm 6$ years. The sequences were acquired at rest and during submaximal bicycle exercise (at a heart rate of 100-110 beats per minute, before fusion of the early and late diastolic (atrial) velocities of mitral inflow) [4]. Velocity patterns were extracted from myocardial velocity acquisitions, using a fixed sample (size $1 \times 10 \mathrm{~mm}$, located $\approx$ $10 \mathrm{~mm}$ above the mitral annulus at end-systole) at the basal septum and basal lateral wall of the left ventricle (LV). We consider that these regions are sufficient to account for the global longitudinal changes possibly present in the ventricles of the studied HFPEF subjects. The samples were kept fixed to minimize the variability that may appear when tracking the measured regions along the heart cycle, thus maintaining the acquisition as simple as possible. Case-per-case examination was performed to check that the sample area remained within the myocardial wall. The total number of extracted features was 4 (septal/lateral at rest/submaximal), referred to as global analysis, extendible to 16 for a local analysis, where different cardiac phases are treated independently (systole, isovolumic relaxation, early and late diastole). An example of the data extracted for a given subject is illustrated in Fig.2. Class labels based on clinical diagnosis were provided together with the database. However, the subsequent learning was 
(a)

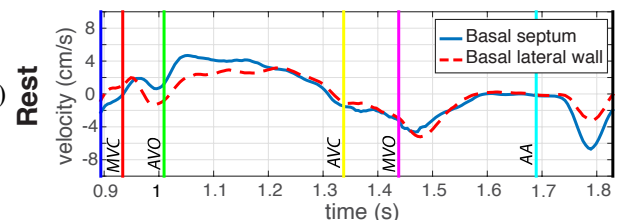

(b)
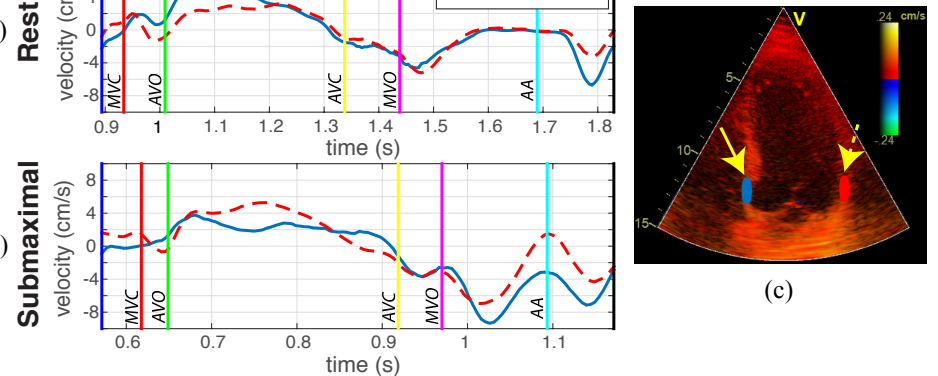

(c)

Fig. 2: Velocity patterns at rest (a) and submaximal (b) stages, corresponding to the snapshot (c) of a myocardial velocity acquisition from a sample subject. The patterns correspond to the basal septum and lateral wall regions, indicated by arrows in (c). Depicted physiological events: MVC (mitral valve closure), AVO (aortic valve opening), AVC (aortic valve closure), MVO (mitral valve opening) and AA (atrial activation).

performed in an unsupervised way. Such labels were only used to compare the characterization of the learnt representation.

Temporal normalization. Subjects differ in terms of intrinsic heart rate and timing of cardiac phases (e.g. systole, diastole). This is even more pronounced between the rest and submaximal sequences of a given subject. In order to consistently compare subjects, their measurements need to match temporally. To this end, a two-stage normalization was applied. The first stage consisted in a piece-wise linear warping of the timescale, using physiological events normalized to the heart cycle. These events were: start and end of the heart cycle (onset of QRS on the ECG), mitral/aortic valves opening and closure (identified from Doppler flows, acquired separately but at similar heart rates) and atrial activation (onset of $\mathrm{P}$-wave on the ECG). The timescales were redefined towards a common reference. This reference was selected from the healthy controls, as the one with the most central velocity patterns across a range of tests where each subject was considered successively as reference. The most central pattern is the one for which the sum of Euclidean distances to the remaining patterns is minimized. Then, after achieving a common temporal reference, the second stage consisted in resampling the velocity data to the new common temporal reference, through spline interpolation [2].

\subsection{Parameters tuning}

The bandwidth $\sigma_{m}$ of the kernel $\mathbf{K}_{m}$ was calculated feature-wise as the average of the pairwise Euclidean distances between each sample and its $k$-th nearest neighbor (looking at the corresponding feature). In this case $k=11$. Then, the number of neighbors used to define the affinity matrix was fixed to 4 . These two values were established heuristically, aiming at maximizing the spread of each 

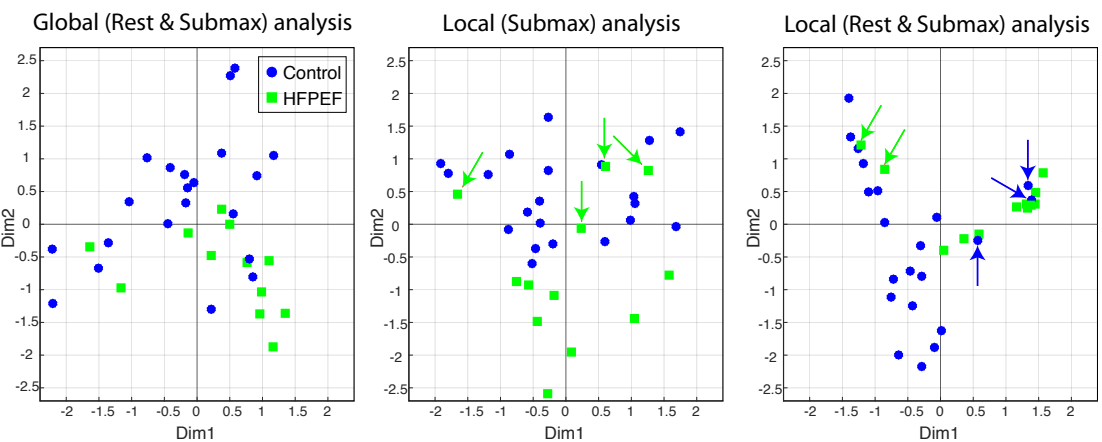

Fig. 3: Three examples of output space: (a) is the result of a global analysis, including rest and submaximal stages, (b) corresponds to a local analysis at submaximal stage and (c) is obtained by a local analysis at rest and submaximal stages jointly. When clusters are intuited, "misclassified" subjects are pointed with arrows.

class in the output space. Last, the number of iterations of the optimization was determined according to the convergence rate. In our implementation, it was fixed to 3 iterations, since at this point the algorithm had converged to a final solution (noticed by the asymptotic decrease of the difference between the optimized variables among consecutive iterations). Unlike approaches using large databases of very different images [8], we think that the relative similarity of the studied features (physiological data in a coherent population) may contribute to this fast convergence.

\subsection{Algorithm output and discussion of the results}

Learnt representation From the $N$-dimensional output space provided by the MKL algorithm, the two first dimensions (associated to the largest eigenvalues) are kept to form a representation that reduces the dimensionality of the complex ambient space and that is consistent with the phenomenon under study. In this particular application, we search to characterize the HFPEF syndrome, which mainly presents diastolic abnormalities in the LV. In an ideal representation, subjects would be mapped class-wise and clear clinically-defined clusters would stand out. Previous works [5] specifically target the recovery of a clustered space by integrating support vector machine techniques to the MKL framework. In contrast, in our application, the uncertainty about the definition of clear clinical clusters is high. Thus, we prefer to keep the problem unsupervised. This also leaves the door open for further exploitation of the data distribution in the output space, such as variability analysis or metrics computation.

Features combination We evaluated the contribution of using multiple descriptors consistent with the disease, against a single descriptor approach. Global and local temporal analyses were performed, taking into account the entire pattern or temporal windows corresponding to different cardiac phases, respectively. 
Figure 3 provides an example of three output spaces obtained by: (a) global analysis including rest and submaximal stages, (b) local analysis at submaximal stage, and (c) local analysis fusing rest and submaximal stages. In these experiments, septal and lateral regions were jointly analyzed, corresponding to rows 11, 10 and 12 in Tab.1, respectively. This table summarizes some tested configurations with the available data.

Suggested labeling The number of "misclassified" subjects (second-to-last column in Tab.1) serves as an indicator of the accuracy of the representation characterizing the HFPEF syndrome. It is qualitatively determined by visual inspection of the learnt representation by: first, recognizing class-related clusters from the output space; then, identifying subjects lying in a "wrong" cluster based on the clinical labels (indicated by arrows in Fig.3). Empty values in some experiments indicate the absence of distinguishable regions within the representation. To support the qualitative interpretation, sensitivity and specificity were calculated (last column in Tab.1), considering clinical decision as the gold standard. Notice that the highest sensitivity corresponds to the most exhaustive analysis.

The results of Tab.1 suggest that a local analysis is more accurate than a global analysis in all cases. By splitting the pattern into physiological temporal windows (systole, iso-volumic relaxation, early and late diastole), the weights estimated by the algorithm point out their relative importance (early diastole the most prominent), which coincides with the physiological features of interest discussed in the literature [4]. Besides, by analyzing jointly different regions (basal septum and basal lateral wall) and different stages (rest and submaximal) the obtained representation is more explanatory. Better defined regions emerge, consistently with the class provided with the database. Finally, we observed that analyzing basal lateral wall alone was not sufficient to differentiate healthy from diseased subjects (for this reason these experiments are not shown in Tab.1).

\begin{tabular}{|c|c|c|c|c|c|}
\hline Test & Region & Stage & Analysis & Misc. & Sens./Spec. (\%) \\
\hline 1 & Septal & Rest & Global & - & - \\
\hline 2 & Septal & Rest & Local & - & - \\
\hline 3 & Septal & Submax & Global & - & - \\
\hline 4 & Septal & Submax & Local & - & - \\
\hline 5 & Septal & Rest + Submax & Global & 7 & $50.0 / 95.2$ \\
\hline 6 & Septal & Rest + Submax & Local & 6 & $58.3 / 95.2$ \\
\hline 7 & Septal + Lateral & Rest & Global & - & - \\
\hline 8 & Septal + Lateral & Rest & Local & 6 & $66.6 / 90.5$ \\
\hline 9 & Septal + Lateral & Submax & Global & - & - \\
\hline 10 & Septal + Lateral & Submax & Local & 4 & $66.6 / 100.0$ \\
\hline 11 & Septal + Lateral & Rest + Submax & Global & - & - \\
\hline 12 & Septal + Lateral & Rest + Submax & Local & 5 & $83.3 / 85.7$ \\
\hline
\end{tabular}

Table 1: Different launches tested on the available data. Symbol "." indicates the absence of distinguishable regions within the representation. "Misc." stands for misclassified and "Sens./Spec." stand for sensitivity and specificity. 
Clinical potential According to the requirements of clinical practice, the algorithm organizes the data such that a grading appears between healthy and diseased subjects. Referring to the best representation (Fig.3c and last row in Tab.1), the "misclassified" subjects pointed with green arrows were confirmed to be outliers by a clinically experienced observer. These subjects presented clinical conditions different from HFPEF, which were found to be: left bundle branch block (LBBB), right ventricular dysfunction (that affects the LV). These conditions may or may not be reflected in the velocity pattern, thus to characterize them properly, meaningful complementary descriptors should be added to the study. It is worth mentioning that although experiment 10 (Tab.1) seems to be the best in terms of "misclassified" subjects, two of them do not lie in their corresponding region (being false negatives). On the contrary, no significant class-wise differences based on E/e' were reported in the literature [4].

\section{Conclusions}

We presented a method for quantitatively characterizing myocardial velocities in a population. Its major strength lies in its ability to jointly analyze information, which is demonstrated to improve its explanatory capability. In the context of HFPEF, the method succeeded in differentiating healthy from diseased subjects, which contrasts with the poor performance of simpler analyses, such as those currently led in the clinical practice involving E/e' ratio. By generating hypotheses for discriminative variables, the method has high potential to reach a better understanding of the HFPEF syndrome, which is highly challenging due to its heterogeneity. Perspectives for the method consist of taking advantage of the lower-dimensional representation to quantitatively support clinical diagnosis on a broader dataset.

Acknowledgements. The authors acknowledge European Union 7th Framework Programme (VP2HF FP7-2013-611823) and the Spanish Ministry of Economy and Competitiveness (TIN2012-35874). The work of S. Sanchez-Martinez was supported by a fellowship from "la Caixa" Banking Foundation.

\section{References}

1. Belkin, M., Niyogi, P.: Laplacian eigenmaps for dimensionality reduction and data representation. MIT Press 15, 1373-1396 (2003)

2. Duchateau, N., De Craene, M., Piella, G., et al.: A spatiotemporal statistical atlas of motion for the quantification of abnormal myocardial tissue velocities. Med Image Anal 15, 316-328 (2011)

3. Duchateau, N., De Craene, M., Piella, G., et al.: Constrained manifold learning for the characterization of pathological deviations from normality. Med Image Anal 16, 1532-1549 (2012)

4. Erdei, T., Smiseth, O., Marino, P., et al.: A systematic review of diastolic stress tests in heart failure with preserved ejection fraction, with proposals from the EU-FP7 MEDIA study group. Eur J Heart Fail 16, 1345-1361 (2014) 
5. Gönen, M.: Supervised multiple kernel embedding for learning predictive subspaces. IEEE Trans Knowl Data Eng 25, 2381-2389 (2013)

6. Grant, M., Boyd, S.: CVX: Matlab software for disciplined convex programming, version 2.0 beta. http://cvxr.com/cvx (September 2013)

7. Komajda, M., Lam, C.: Heart failure with preserved ejection fraction: a clinical dilemma. Eur Heart J 35, 1022-1032 (2014)

8. Lin, Y., Liu, T., Fuh, C.: Multiple kernel learning for dimensionality reduction. IEEE Trans Pattern Anal Mach Intell 33, 1-14 (2011)

9. Paulus, W., Tschöpe, C., Sanderson, J., et al.: How to diagnose diastolic heart failure: a consensus statement on the diagnosis of heart failure with normal left ventricular ejection fraction by the Heart Failure and Echocardiography Associations of the European Society of Cardiology. Eur Heart J 28, 2539-2550 (2007)

10. Senni, M., Paulus, W., Gavazzi, A., et al.: New strategies for heart failure with preserved ejection fraction: the importance of targeted therapies for heart failure phenotypes. Eur Heart J pp. 2797-2811 (2014)

11. Yan, S., Xu, D., Zhang, B., et al.: Graph embedding and extensions: a general framework for dimensionality reduction. IEEE Trans Pattern Anal Mach Intell 29, 40-51 (2007) 